\title{
A NOTE ON THE TIME SERIES MEASURE OF CONSERVATISM
}

\section{Sebastian Brauer and Frank Westermann}

\author{
Working Paper 81
}

February 2010

INSTITUTE FOR EMPIRICAL ECONOMIC RESEARCH

University of Osnabrück

Rolandstraße 8

49069 Osnabrück

Germany 


\title{
A note on the time series measure of conservatism*
}

\author{
Sebastian Brauer \\ Institute for Empirical Economic Research \\ University of Osnabrueck \\ Germany \\ and \\ Frank Westermann ${ }^{\dagger}$ \\ Institute for Empirical Economic Research \\ University of Osnabrueck \\ Germany \\ and CESifo
}

February 17, 2010

\footnotetext{
${ }^{*}$ We would like to thank Sudipta Basu and Joachim Wilde, as well as the seminar participants at UC San Diego and the University of Osnabrueck for helpful comments and suggestions.

${ }^{\dagger}$ Corresponding author: frank.westermann@uni-osnabrueck.de. Institute for Empirical Economic Research. University of Osnabrueck. Rolandstraße 8. 49069 Osnabrueck, Germany. Tel.: ++49-541-969-3508. Fax: $++49-541-969-2757$.
} 


\section{Abstract}

Asymmetric persistence of accounting income is often tested in a regression of changes in earnings on lagged changes in earnings, including an interaction term for negative changes (see Basu [1997] or Ball et al. [2009] for a recent overview). In this note we propose an alternative, but closely related measure of conservatism - regressing the changes in earnings on the lagged levels, similar to the threshold-unit root test specification of Enders and Granger [1998]. We argue that this approach has three distinct advantages compared to the conventional setup: (i) a smooth, non-oscillating impulse response pattern to an unexpected shock in earnings (ii) a return to the old equilibrium of earnings in the long run and (iii) it can be extended to higher order autoregressive processes. We illustrate the differences between the two approaches, when applied to a common data set of firms, as well as a data set from a Monte Carlo simulation.

JEL Classification: M41, C23

Keywords: Timely loss recognition, Asymmetric persistence, Conservatism 


\section{Introduction}

In this note, we discuss the time series approach of measuring conservatism, one of the four alternatives offered in the seminal paper of Basu $[1997]^{1}$. In this approach, it is argued that negative income changes - as an approximation of negative news - should be less persistent than positive income changes, when anticipating future losses at an early stage.

This hypothesis of asymmetric persistence is often tested in a regression of the changes in earnings on the lagged changes in earnings, including an interaction-term that indicates negative values. The intuition for this setup is the following - if there is mean reversion, then negative changes must be followed by positive changes in subsequent periods. Thus, a negative coefficient in this regression is taken as evidence of low persistence of negative news and conservatism.

We start our discussion of this setup by pointing out that a negative coefficient in this regression implies an oscillating impulse response pattern to a standard shock in earnings, a property of the regression that so far has not yet been considered in the literature. This is because news are not only contained in the change in income, but also in the error term of the regression ${ }^{2}$. As the coefficient measures the systematic link between current and lagged changes, an unexpected negative shock - a bad draw in the otherwise mean zero error term does not only lead to a positive reaction in the following period, but also to another negative change in the period after that, a positive one in the next year, and so on. From period to period, reactions with alternating signs get smaller and smaller and eventually converge to zero.

We furthermore argue that in this specification it is not possible to fully identify transitory vs. persistent components in earnings, as a negative coefficient does not ensure that the time series in levels eventually converges back to its old steady state. We illustrate that accumulating all the positive and negative changes over time can lead to a new long run equilibrium, even when the coefficient is negative and large. Although a negative correlation of growth rates is a first sign of the initial mean reversion, it is not informative about the medium and long run reaction to shocks.

As an alternative approach, we suggest that a negative coefficient in a regression of changes on the lagged levels of earnings indeed implies a smooth (non-oscillating) transition back to the steady state. In this approach, a negative coefficient also ensures that shocks are transitory when taking a medium and long term perspective. When adding an interaction term for

\footnotetext{
${ }^{1}$ This approach has been used recently in several academic papers. See Ball et al. [2003], Ball and Shivakumar [2005], or Ball et al. [2009] for a discussion on the measurement of conservatism.

${ }^{2}$ With R-squares of often less than $10 \%$ in the Basu regression, the analysis of such unexpected changes clearly seems to be of high relevance to the overall analysis.
} 
negative values, it is related to the Enders and Granger [1998] threshold autoregressive (TAR) model. The impulse response pattern to an unexpected negative shock in this regression is closely related to the one-time dip in the earnings process that is displayed in Figure 1b of Basu [1997] and we therefore argue that it is conceptually more consistent with the testable hypothesis of conservatism.

Within the threshold unit root approach, it is possible to test for persistence after (i) negative lagged levels (ii) negative lagged changes and (iii) below average values. Choosing the appropriate definition of the dummy variable, it is not subject to the critique of survivorship bias that the literature often attributes to the regression of levels on lagged levels, as it is not necessary to condition on actual losses.

Finally, our proposed regression can easily be extended to higher order autoregressive processes, by including further lagged changes in earnings, just as a standard Dickey-Fuller unit root test can be extended to an augmented Dickey-Fuller test. The augmented Dickey-Fuller test is often applied in empirical macroeconomics, when conducting tests of persistence in aggregate income (see Nelson and Plosser [1982], Campbell and Mankiw [1987] and Cheung and Chinn [1997]).

We apply both approaches to a common data set of 136 firms in the S\&P500 index that were listed continuously from 1950 until 2008. In our alternative regression setup, we can confirm the main findings in the literature on the asymmetric timeliness of loss recognition, in particular the asymmetry between positive and negative shocks. We also find, however, that the results differ in the details of the rest of the regression. For instance, the response of the positive shock in our specification is also transitory, although the return to steady state takes much longer than in the case of a negative shock - a result that appears quite plausible in the context of the underlying assumption on the transitory nature of accounting income.

In order to evaluate why our alternative approach overall leads to qualitatively similar findings as the previous literature, we conduct a Monte Carlo simulation, where we simulate several AR(1) time series, with different degrees of persistence. The advantage of this approach is that we know the true data generating process and we can evaluate which regression specification identifies the persistence levels of this process more precisely. We find that for most persistence levels, both regression specifications can identity the mean reversion that is present in the data. The regression of changes on lagged changes, however, substantially underestimates the true degree of mean reversion. Furthermore, for persistence levels close to unity, the unit root test still is able to identify mean reversion, while in the regression of changes on lagged changes, the reversion coefficient becomes insignificant. This simulation helps to explain the findings on positive shocks in the data set of the S\&P500 firms. 


\section{The time series measure of conservatism}

In the Basu [1997] and Ball and Shivakumar [2005] time series approach to measuring conservatism, economic income is assumed to be completely transitory and independent of prior periods, whereas accounting income depends on prior periods through the delayed translation into the accounts. These and related papers ${ }^{3}$ have aimed to document, that under conservative behavior, negative changes in income are more transitory than positive changes.

The regression specification that is typically used to test this hypothesis is:

$$
\Delta N I_{i, t}=\alpha_{0}+\alpha_{1} D_{i, t-1}+\alpha_{2} \Delta N I_{i, t-1}+\alpha_{3} D_{i, t-1} * \Delta N I_{i, t-1}+\epsilon_{i, t}
$$

where $\Delta N I_{i, t}$ is the change in net income standardized with totals assets (from $t-1$ ), $\Delta N I_{i, t-1}$ is the lagged change in net income, and $D_{i, t-1}$ is a dummy variable that takes the value of one if the lagged change was negative and zero otherwise. $\epsilon_{i, t}$ is a mean zero error term. The standard interpretation is the following: $\alpha_{2}=0$, if deferred recognition of economic gains in accounting income leads to persistence of positive income shocks. $\alpha_{2}+\alpha_{3}<0$ if economic losses are transitory components in accounting income. Concerning conservatism, $\alpha_{3}<0$, if losses are recognized more timely than gains. The intuition for the Basu [1997] and Ball and Shivakumar [2005] test specification appears straightforward. If income is to be transitory, positive changes need to be followed by negative changes in later periods.

Our first main point in this note is that the test specification in equation 1 is to rigid to test this straightforward intuition. This can be best illustrated, when neglecting the focus on gains and losses for a moment and simply looking at the stochastic process that drives income in general over time. In this case the regression specification - without the dummy and the interaction term - can be simplified to:

$$
\Delta N I_{i, t}=\alpha_{0}+\alpha_{1} \Delta N I_{i, t-1}+\epsilon_{i, t}
$$

A negative and significant $\alpha_{1}$ in regression 2, following the Basu [1997] intuition, would be consistent with the view that income is transitory and the larger the negative coefficient, the faster the mean reversion in income. However, negative changes in income would not only be followed by positive changes in period $t+1$, but also by another negative jump in $t+2$, again a positive jump in the following year, and so on. If $\alpha_{1}$ is negative it would imply an oscillating impulse response pattern to a standard shock in income. The impulse response pattern implied by a coefficient of, for instance, -0.692 (the baseline estimate in Basu [1997]), is indicated by the red solid line (marked by triangles) in Figure 1. This oscillating impulse response pattern is difficult to be reconciled with the hypothesis behind the Basu [1997] setup - a single period negative downturn for negative income shocks, as outlined in Figure 1b of

\footnotetext{
${ }^{3}$ See Section 6 .
} 


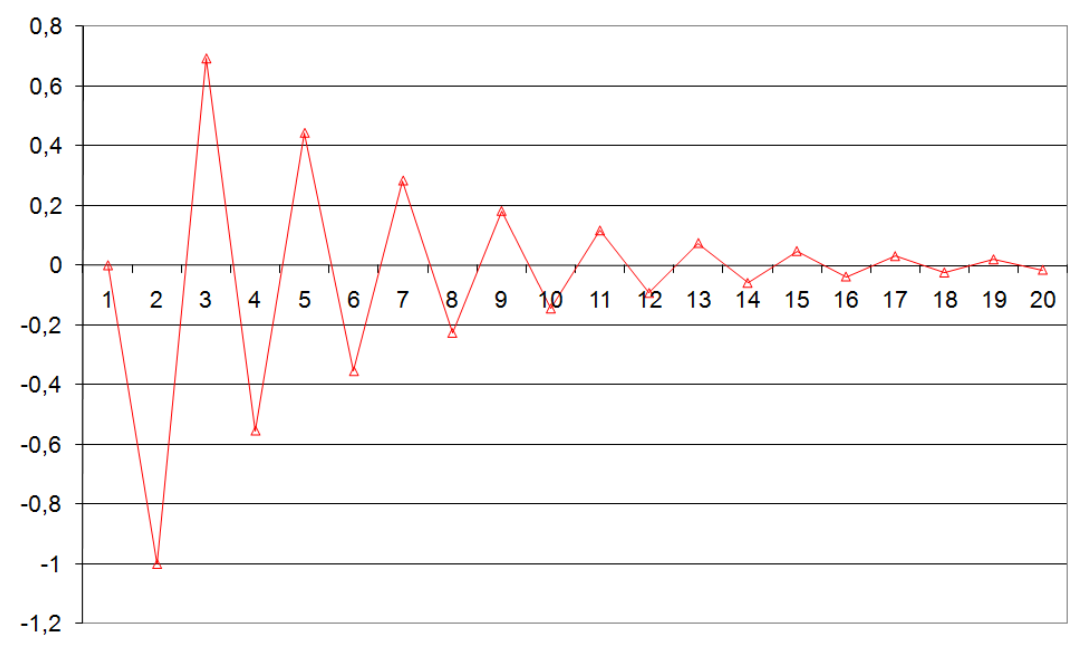

Figure 1: Impulse response pattern of positive and negative alpha, with alpha $<1$, in the Basu [1997] regression

his paper.

This point can also be made more formally, by inverting the $\mathrm{AR}(1)$ process to a moving average (MA) process. We start from equation 2, ignoring the constant and using the fact that the time series process is stable over time. We then can recursively insert values for $\Delta N I_{i, t-1}$ :

$$
\begin{aligned}
\Delta N I_{i, t}= & \alpha_{1} \Delta N I_{i, t-1}+\epsilon_{i, t} \\
= & \alpha_{1}\left(\alpha_{1} \Delta N I_{i, t-2}+\epsilon_{i, t-1}\right)+\epsilon_{i, t} \\
= & \alpha_{1}^{2} \Delta N I_{i, t-2}+\alpha_{1} \epsilon_{i, t-1}+\epsilon_{i, t} \\
= & \alpha_{1}^{2}\left(\alpha_{1} \Delta N I_{i, t-3}+\epsilon_{i, t-2}\right)+\alpha_{1} \epsilon_{i, t-1}+\epsilon_{i, t} \\
& \cdots \\
= & \alpha_{1}^{n} \Delta N I_{i, t-n}+\alpha_{1}^{n-1} \epsilon_{i, t-n-1}+\ldots+\alpha_{1}^{2} \epsilon_{i, t-2}+\alpha_{1} \epsilon_{i, t-1}+\epsilon_{i, t},
\end{aligned}
$$

and, letting $n$ go to infinity, we find that:

$$
\operatorname{Lim}_{n \rightarrow \infty} \Delta N I_{i, t}=\sum_{n=1}^{\infty} \alpha_{1}^{n} \epsilon_{i, t-n}+\epsilon_{i, t} .
$$

In this MA representation of the $\mathrm{AR}(1)$ process, we see that for a negative $\alpha$, the response to an unanticipated shock must oscillate, as the coefficient is raised to the $n$-th power. Thus, there will be positive reactions in even years and negative reactions in odd years.

When we accumulate these changes, in order to get back to the levels of income - that are more directly comparable to the time paths displayed in Figure 1b of Basu [1997] - the amplitude of the oscillating pattern decreases somewhat, but a second problem of the specification becomes apparent. As illustrated in Figure 2, there remains a substantial positive persistent 
component in the long run. The long run steady state persistence level can be computed as $\frac{1}{1-\alpha_{1}}$. The negative coefficient of $\alpha_{1}$ is therefore not sufficient to conclude that the changes in income are not persistent in the long run.

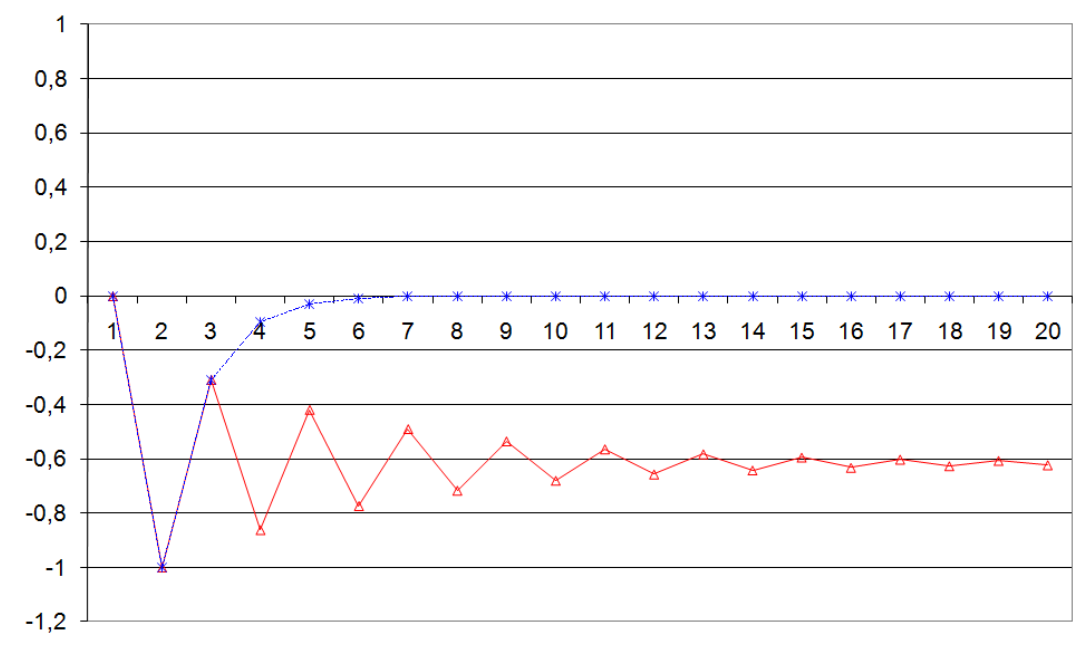

Figure 2: Accumulation of changes

\section{An alternative approach}

A more conventional time series test for persistence would regress the changes in a given variable on the lagged levels, rather than on the lagged changes. This specification, often referred to as the Dickey and Fuller [1979, 1981] test is discussed, for instance, in Hamilton [1994], or Luetkepohl and Kraetzig [2004], and is widely used in applied time series econometrics. In this section we would like to illustrate how conservatism could be tested in this alternative setup.

Suppose we start from a regression similar to equation 2, but with the levels of income, $N I_{i, t-1}$, on the right hand side, rather than the changes, $\Delta N I_{i, t-1}{ }^{4}$ :

$$
\Delta N I_{i, t}=\beta_{0}+\beta_{1} N I_{i, t-1}+\epsilon_{i, t}
$$

The estimation of the coefficient $\beta_{1}$ has several benefits compared to the estimation of $\alpha_{1}$ in regression 2. First, a negative coefficient on $\beta_{1}$ would imply the smooth impulse response pattern shown in the blue line (marked by stars) of Figure 2 that comes much closer to the time path after a negative shock displayed in Figure 1b of Basu $[1997]^{5}$. The larger the $\beta_{1}$, the

\footnotetext{
${ }^{4}$ This specification is abstracting from serial correlation in the changes of accounting income. This lagged terms could be added to the regression as control variables, without changing the interpretation of the $\beta_{1}$ coefficient. This would than be a full augmented Dickey and Fuller [1979] test specification as discussed in Hamilton [1994], equation 17.7.11.

${ }^{5}$ Formally, this can be shown, when adding $N I_{i, t-1}$ on both sides of the equation. As $\beta_{1}+1$ is always positive,
} 
faster the reversion to the mean. If $\beta_{1}$ is equal to zero, changes in income would be persistent, just like in the interpretation for $\alpha_{1}$ in the original Basu [1997] and Ball and Shivakumar [2005] regression. Furthermore, if $\beta_{1}<0$, it would imply that in the long run the persistence of the shocks would actually be equal to zero: When the lagged levels were small, the change would be positive and if lagged levels were large, changes would be negative. A negative coefficient of $\beta_{1}$ would therefore imply the return to a long run steady state, and any deviation from this steady state would be transitory.

In an extension of this simplified regression, one could estimate the following equation that addresses the issue of asymmetric persistence of negative and positive gains in income data, when adding an interaction term, $D_{i, t-1}$, that captures negative lagged values:

$$
\Delta N I_{i, t}=\beta_{0}+\beta_{1} D_{i, t-1}+\beta_{2} N I_{i, t-1}+\beta_{3} D_{i, t-1} * N I_{i, t-1}+\epsilon_{i, t} .
$$

In this regression, positive income gains would be persistent if $\beta_{2}=0$. Negative income would be transitory if $\beta_{2}+\beta_{3}<0$ and losses would be recognized more timely than gains if $\beta_{3}<0$.

The regression equation in 4 is related to the unit root tests with asymmetric adjustment proposed by Enders and Granger [1998]. In their threshold autoregressive (TAR) models, they test for the same type of asymmetric persistence after positive and negative lagged values, or changes - in an application to the term structure of interest rates - as the empirical accounting literature does in earnings data. The next section shows that a direct estimation of a TAR model leads to similar results as regression 4 , that remains more closely related to the original Basu specification. ${ }^{6}$

The dummy variable can take different forms in the TAR model and in our proposed setup. It is possible to test the persistence of shocks after negative lagged levels, negative lagged changes, and above (or below) average values. In the empirical section, we will discuss all three possibilities.

An advantage of the TAR approach is that it can be easily extended to higher order autoregressive processes. Suppose the true data generating process is an $\operatorname{AR}(p)$, rather than an $\mathrm{AR}(1)$ process. In this case, the regression can be augmented with lagged values of the changes in income (a derivation of this specification is fully analogous to the derivation of the augmented Dickey-Fuller test). The specification of higher order autoregressive processes

\footnotetext{
and between 0 and 1 , any change in $\epsilon_{i, t}$ would smoothly disappear over time.

${ }^{6}$ Note that the TAR model was not yet developed at the time of the original contribution of Basu [1997]. We also do not fully adopt the TAR model, as in principle the Basu [1997] regression is a more natural setup for the research question of conservatism. While the TAR model tests whether positive and negative shocks, respectively, are persistent, the Basu [1997] setup is designed to test the significance of difference in the persistence between the two. This later question could only be answered in the Enders and Granger [1998] TAR model, when additionally performing a Wald test.
} 
would be :

$$
\Delta N I_{i, t}=\beta_{0}+\beta_{1} D_{i, t-1}+\beta_{2} N I_{i, t-1}+\beta_{3} D_{i, t-1} * N I_{i, t-1}+\sum_{j=1}^{p} \gamma_{j} \Delta N I_{i, t}+\epsilon_{i, t} .
$$

In this augmented setup, the interpretation of the coefficients $\beta_{1}, \beta_{2}$ and $\beta_{3}$ would remain unchanged, compared to the regression in equation 4.

\section{An application to a common data set}

In this section we apply both regression specifications to a common data set. We use 136 firms from the S\&P500 index in a sample from 1950 to 2008. This selection contains all firms that have been included in the index since it was first constructed in 1950. We have, thus, a panel data set that can be analyzed without major concerns about some of the other common econometric issues, such as unbalanced samples or small sample properties of the estimators.

We start by estimating the standard Basu [1997] and Ball and Shivakumar [2005] regression (see equation 1). A dummy variable captures whether the changes in the previous period were negative or not. Table 1 shows that we obtain very similar results to those that are typically reported in the literature. In the first set of three columns we report the results for income before extraordinary items, using various techniques of accounting for firm fixed effects. Regression (1) includes no fixed effects. Regression (2) includes firm fixed effects and regression (3) uses the Arellano and Bond [1991] approach that accounts for fixed effects by differencing both sides of the equation ${ }^{7}$. In columns $4-6$, we repeat the exercise for net income. Finally, in columns 7-12, we estimate the same regressions with a panel data set that was corrected for outliers using the multivariate Hadi [1994] procedure, while in regressions $1-6$, we simply excluded the $1 \%$ extreme observations.

In all regressions we confirm the main Basu [1997] and Ball and Shivakumar [2005] results for our data set. In particular, $\alpha_{3}$ is negative and statistically significant, confirming the asymmetric timeliness of earnings. The evidence on $\alpha_{2}$ is more mixed. In five out of twelve regressions, $\alpha_{2}$ is negative and significant, in the remaining regressions it is insignificant. In all cases the coefficients are close to zero. This mixed evidence is also consistent with other studies, but it constitutes a puzzle, when thinking about the implications for the long run. An insignificant coefficient would imply that shocks to positive changes in net income are truly persistent - a positive change in net income would forever remain in the data series. A negative coefficient would imply that positive changes are transitory, but just relatively more persistent than negative changes. Using a Wald Test, we confirm that the sum of $\alpha_{2}$ and $\alpha_{3}$ is negative and significant in all cases.

\footnotetext{
${ }^{7}$ We also tested for Year and Firm/Year fixed effects. The results do not change quantitatively and are available upon request.
} 
Table 1: Regression of changes in earnings on lagged changes in earnings for all firm-years (Basu [1997] specification)

\begin{tabular}{|c|c|c|c|c|c|c|c|c|c|c|c|c|}
\hline \multicolumn{13}{|c|}{$\Delta N I_{i, t}=\alpha_{0}+\alpha_{1} D_{i, t-1}+\alpha_{2} \Delta N I_{i, t-1}+\alpha_{3} D_{i, t-1} * \Delta N I_{i, t-1}+\epsilon_{i, t}$} \\
\hline & - & $\mathrm{Fi}$ & $\mathrm{AB}$ & - & $\mathrm{Fi}$ & $\mathrm{AB}$ & - & $\mathrm{Fi}$ & $\mathrm{AB}$ & - & $\mathrm{Fi}$ & $\mathrm{AB}$ \\
\hline & 1 & 2 & 3 & 4 & 5 & 6 & 7 & 8 & 9 & 10 & 11 & 12 \\
\hline$\alpha_{0}$ & $\begin{array}{l}-0.001 \\
(-1.76)\end{array}$ & $\begin{array}{l}-0.001 \\
(-2.55)\end{array}$ & $\begin{array}{l}-0.003 \\
(-1.94)\end{array}$ & $\begin{array}{l}-0.001 \\
(-1.42)\end{array}$ & $\begin{array}{l}-0.002 \\
(-2.01)\end{array}$ & $\begin{array}{l}-0.004 \\
(-2.19)\end{array}$ & $\begin{array}{l}-0.001 \\
(-1.86)\end{array}$ & $\begin{array}{l}-0.001 \\
(-2.77)\end{array}$ & $\begin{array}{l}-0.002 \\
(-1.25)\end{array}$ & $\begin{array}{l}-0.001 \\
(-2.00)\end{array}$ & $\begin{array}{l}-0.002 \\
(-2.57)\end{array}$ & $\begin{array}{l}-0.003 \\
(-1.41)\end{array}$ \\
\hline$\alpha_{1} D_{i, t-1}$ & $\begin{array}{l}-0.004 \\
(-4.46)\end{array}$ & $\begin{array}{l}-0.004 \\
(-4.58)\end{array}$ & $\begin{array}{l}-0.002 \\
(-1.17)\end{array}$ & $\begin{array}{l}-0.006 \\
(-6.15)\end{array}$ & $\begin{array}{l}-0.006 \\
(-6.28)\end{array}$ & $\begin{array}{l}-0.004 \\
(-1.86)\end{array}$ & $\begin{array}{l}-0.005 \\
(-5.21)\end{array}$ & $\begin{array}{l}-0.005 \\
(-4.95)\end{array}$ & $\begin{array}{l}-0.003 \\
(-1.82)\end{array}$ & $\begin{array}{l}-0.006 \\
(-6.27)\end{array}$ & $\begin{array}{l}-0.007 \\
(-6.42)\end{array}$ & $\begin{array}{l}-0.005 \\
(-2.83)\end{array}$ \\
\hline$\alpha_{2} \Delta N I_{i, t-1}$ & $\begin{array}{l}-0.063 \\
(-1.91)\end{array}$ & $\begin{array}{l}-0.047 \\
(-1.58)\end{array}$ & $\begin{array}{l}0.101 \\
(1.27)\end{array}$ & $\begin{array}{l}-0.142 \\
(-4.10)\end{array}$ & $\begin{array}{l}-0.123 \\
(-3.57)\end{array}$ & $\begin{array}{l}0.009 \\
(0.13)\end{array}$ & $\begin{array}{l}-0.065 \\
(-2.20)\end{array}$ & $\begin{array}{l}-0.050 \\
(-1.90)\end{array}$ & $\begin{array}{l}0.057 \\
(0.63)\end{array}$ & $\begin{array}{l}-0.137 \\
(-4.45)\end{array}$ & $\begin{array}{l}-0.118 \\
(-3.67)\end{array}$ & $\begin{array}{l}-0.056 \\
(-0.68)\end{array}$ \\
\hline$\alpha_{3} D_{i, t-1} * \Delta N I_{i, t-1}$ & $\begin{array}{l}-0.206 \\
(-4.47) \\
\end{array}$ & $\begin{array}{l}-0.244 \\
(-5.08)\end{array}$ & $\begin{array}{l}-0.351 \\
(-2.55)\end{array}$ & $\begin{array}{l}-0.261 \\
(-5.32)\end{array}$ & $\begin{array}{l}-0.305 \\
(-5.21)\end{array}$ & $\begin{array}{l}-0.395 \\
(-3.41)\end{array}$ & $\begin{array}{l}-0.246 \\
(-5.91)\end{array}$ & $\begin{array}{l}-0.281 \\
(-6.68) \\
\end{array}$ & $\begin{array}{l}-0.313 \\
(-1.96)\end{array}$ & $\begin{array}{l}-0.282 \\
(-6.16) \\
\end{array}$ & $\begin{array}{l}-0.326 \\
(-5.71) \\
\end{array}$ & $\begin{array}{l}-0.328 \\
(-2.40)\end{array}$ \\
\hline Obs. & 7,368 & 7,368 & 7,368 & 7,364 & 7,364 & 7,364 & 7,428 & 7,428 & 7,428 & 7,412 & 7,412 & 7,412 \\
\hline$R^{2}$ & 0.022 & 0.022 & - & 0.056 & 0.056 & - & 0.031 & 0.031 & - & 0.062 & 0.062 & - \\
\hline
\end{tabular}

Definition of variables: $\Delta I X_{i, t}$, change in income before extraordinary items for firm $i$ from year $t-1$ to year $t$ after standard outlier detection. $\Delta N I_{i, t}$, change in net income for firm $i$ from year $t-1$ to year $t$ after standard outlier detection. $\Delta I X H_{i, t}$, change in income before extraordinary items for firm $i$ from year $t-1$ to year $t$ after outlier detection by Hadi [1994]. $\Delta N I H_{i, t}$, change in net income for firm $i$ from year $t-1$ to year $t$ after outlier detection by Hadi [1994]. $D_{i, t-1}=1$ if $\Delta N I_{i, t-1}<0 ;=0$ otherwise. All variables are standardized by total assets for firm $i$ at the end of year $t-1$.

The regressions exclude extreme $1 \%$ on each side in the standard outlier detection. The outlier detection by Hadi [1994] correspondently contains a significance level of $1 \%$.

White [1980] t-statistics in parentheses for the regressions with and without fixed effects. Windmeijer [2005] corrected z-statistics in parentheses for the regressions with the Arellano and Bond [1991] estimator. 
Table 2: Regression of changes in earnings on lagged levels of earnings for all firm-years

\begin{tabular}{|c|c|c|c|c|c|c|c|c|c|c|c|c|}
\hline \multicolumn{13}{|c|}{$\Delta N I_{i, t}=\beta_{0}+\beta_{1} D_{i, t-1}+\beta_{2} N I_{i, t-1}+\beta_{3} D_{i, t-1} * N I_{i, t-1}+\epsilon_{i, t}$} \\
\hline & \multicolumn{3}{|c|}{$\Delta I X_{i, t}$} & \multicolumn{3}{|c|}{$\Delta N I_{i, t}$} & \multicolumn{3}{|c|}{$\Delta I X H_{i, t}$} & \multicolumn{3}{|c|}{$\Delta N I H_{i, t}$} \\
\hline & - & $\mathrm{Fi}$ & $\mathrm{AB}$ & - & $\mathrm{Fi}$ & $\mathrm{AB}$ & - & $\mathrm{Fi}$ & $\mathrm{AB}$ & - & $\mathrm{Fi}$ & $\mathrm{AB}$ \\
\hline & 1 & 2 & 3 & 4 & 5 & 6 & 7 & 8 & 9 & 10 & 11 & 12 \\
\hline \multicolumn{13}{|l|}{ Panel (a) $\Delta N I_{i, t-1}<0$} \\
\hline$\beta_{0}$ & $\begin{array}{c}0.008 \\
(10.05)\end{array}$ & $\begin{array}{c}0.017 \\
(12.61)\end{array}$ & $\begin{array}{l}0.029 \\
(7.66)\end{array}$ & $\begin{array}{l}0.009 \\
(8.24)\end{array}$ & $\begin{array}{c}0.018 \\
(11.29)\end{array}$ & $\begin{array}{l}0.030 \\
(9.08)\end{array}$ & $\begin{array}{c}0.009 \\
(10.76)\end{array}$ & $\begin{array}{c}0.018 \\
(14.04)\end{array}$ & $\begin{array}{l}0.024 \\
(8.44)\end{array}$ & $\begin{array}{l}0.009 \\
(8.85)\end{array}$ & $\begin{array}{c}0.018 \\
(11.89)\end{array}$ & $\begin{array}{l}0.024 \\
(7.01)\end{array}$ \\
\hline$\beta_{1} D_{i, t-1}$ & $\begin{array}{l}-0.003 \\
(-4.38)\end{array}$ & $\begin{array}{l}-0.004 \\
(-4.55)\end{array}$ & $\begin{array}{l}-0.004 \\
(-2.44)\end{array}$ & $\begin{array}{l}-0.004 \\
(-4.25)\end{array}$ & $\begin{array}{l}-0.004 \\
(-4.15)\end{array}$ & $\begin{array}{l}-0.004 \\
(-2.25)\end{array}$ & $\begin{array}{l}-0.004 \\
(-5.04)\end{array}$ & $\begin{array}{l}-0.004 \\
(-4.92)\end{array}$ & $\begin{array}{l}-0.005 \\
(-3.15)\end{array}$ & $\begin{array}{l}-0.004 \\
(-4.65)\end{array}$ & $\begin{array}{l}-0.005 \\
(-4.50)\end{array}$ & $\begin{array}{l}-0.005 \\
(-2.68)\end{array}$ \\
\hline$\beta_{2} N I_{i, t-1}$ & $\begin{array}{c}-0.123 \\
(-14.62)\end{array}$ & $\begin{array}{c}-0.229 \\
(-15.05)\end{array}$ & $\begin{array}{l}-0.383 \\
(-8.39)\end{array}$ & $\begin{array}{c}-0.145 \\
(-14.09)\end{array}$ & $\begin{array}{c}-0.260 \\
(-14.45)\end{array}$ & $\begin{array}{c}-0.409 \\
(-10.78)\end{array}$ & $\begin{array}{c}-0.130 \\
(-16.00)\end{array}$ & $\begin{array}{c}-0.238 \\
(-16.86)\end{array}$ & $\begin{array}{l}-0.305 \\
(-8.66)\end{array}$ & $\begin{array}{c}-0.150 \\
(-15.53)\end{array}$ & $\begin{array}{c}-0.264 \\
(-15.39)\end{array}$ & $\begin{array}{l}-0.328 \\
(-7.66)\end{array}$ \\
\hline$\beta_{3} D_{i, t-1} * N I_{i, t-1}$ & $\begin{array}{l}-0.179 \\
(-5.76)\end{array}$ & $\begin{array}{l}-0.117 \\
(-3.16)\end{array}$ & $\begin{array}{l}-0.071 \\
(-1.76) \\
\end{array}$ & $\begin{array}{l}-0.269 \\
(-8.16)\end{array}$ & $\begin{array}{l}-0.201 \\
(-5.06)\end{array}$ & $\begin{array}{l}-0.138 \\
(-3.12)\end{array}$ & $\begin{array}{l}-0.216 \\
(-7.64)\end{array}$ & $\begin{array}{l}-0.149 \\
(-4.16)\end{array}$ & $\begin{array}{l}-0.083 \\
(-2.06) \\
\end{array}$ & $\begin{array}{l}-0.300 \\
(-9.49)\end{array}$ & $\begin{array}{l}-0.230 \\
(-5.94) \\
\end{array}$ & $\begin{array}{l}-0.167 \\
(-3.34) \\
\end{array}$ \\
\hline Obs. & 7,294 & 7,294 & 7,294 & 7,292 & 7,292 & 7,292 & 7,413 & 7,413 & 7,413 & 7,395 & 7,395 & 7,395 \\
\hline$R^{2}$ & 0.059 & 0.055 & - & 0.086 & 0.081 & - & 0.077 & 0.073 & - & 0.102 & 0.096 & - \\
\hline Panel (b) $N I_{i, t-1}<0$ & & & & & & & & & & & & \\
\hline$\beta_{0}$ & $\begin{array}{l}0.006 \\
(5.21)\end{array}$ & $\begin{array}{c}0.015 \\
(10.14)\end{array}$ & $\begin{array}{l}0.021 \\
(3.98)\end{array}$ & $\begin{array}{l}0.005 \\
(3.80)\end{array}$ & $\begin{array}{l}0.016 \\
(8.08)\end{array}$ & $\begin{array}{l}0.020 \\
(4.25)\end{array}$ & $\begin{array}{l}0.006 \\
(5.90)\end{array}$ & $\begin{array}{c}0.016 \\
(10.99)\end{array}$ & $\begin{array}{l}0.020 \\
(4.48)\end{array}$ & $\begin{array}{l}0.006 \\
(4.63)\end{array}$ & $\begin{array}{l}0.017 \\
(9.19)\end{array}$ & $\begin{array}{l}0.019 \\
(4.76)\end{array}$ \\
\hline$\beta_{1} D N I_{i, t-1}$ & $\begin{array}{l}0.006 \\
(4.40)\end{array}$ & $\begin{array}{l}0.004 \\
(2.45)\end{array}$ & $\begin{array}{l}0.009 \\
(2.56)\end{array}$ & $\begin{array}{l}0.011 \\
(5.82)\end{array}$ & $\begin{array}{l}0.008 \\
(3.36)\end{array}$ & $\begin{array}{l}0.014 \\
(3.56)\end{array}$ & $\begin{array}{l}0.008 \\
(5.29)\end{array}$ & $\begin{array}{l}0.005 \\
(2.78)\end{array}$ & $\begin{array}{l}0.006 \\
(2.04)\end{array}$ & $\begin{array}{l}0.011 \\
(6.38)\end{array}$ & $\begin{array}{l}0.008 \\
(3.50)\end{array}$ & $\begin{array}{l}0.011 \\
(3.30)\end{array}$ \\
\hline$\beta_{2} N I_{i, t-1}$ & $\begin{array}{l}-0.090 \\
(-7.74)\end{array}$ & $\begin{array}{c}-0.200 \\
(-12.53)\end{array}$ & $\begin{array}{l}-0.286 \\
(-4.45)\end{array}$ & $\begin{array}{l}-0.107 \\
(-7.17)\end{array}$ & $\begin{array}{c}-0.232 \\
(-10.84)\end{array}$ & $\begin{array}{l}-0.295 \\
(-5.58)\end{array}$ & $\begin{array}{l}-0.096 \\
(-9.06)\end{array}$ & $\begin{array}{c}-0.212 \\
(-13.70)\end{array}$ & $\begin{array}{l}-0.268 \\
(-4.93)\end{array}$ & $\begin{array}{l}-0.117 \\
(-8.73)\end{array}$ & $\begin{array}{c}-0.245 \\
(-12.64)\end{array}$ & $\begin{array}{l}-0.280 \\
(-5.93)\end{array}$ \\
\hline$\beta_{3} D N I_{i, t-1} * N I_{i, t-1}$ & $\begin{array}{l}-0.087 \\
(-5.14) \\
\end{array}$ & $\begin{array}{l}-0.079 \\
(-4.07) \\
\end{array}$ & $\begin{array}{l}-0.145 \\
(-4.08) \\
\end{array}$ & $\begin{array}{l}-0.122 \\
(-5.82) \\
\end{array}$ & $\begin{array}{l}-0.105 \\
(-4.45) \\
\end{array}$ & $\begin{array}{l}-0.190 \\
(-4.87) \\
\end{array}$ & $\begin{array}{l}-0.097 \\
(-5.98) \\
\end{array}$ & $\begin{array}{l}-0.083 \\
(-4.42) \\
\end{array}$ & $\begin{array}{l}-0.123 \\
(-3.86) \\
\end{array}$ & $\begin{array}{l}-0.122 \\
(-6.21) \\
\end{array}$ & $\begin{array}{l}-0.101 \\
(-4.63) \\
\end{array}$ & $\begin{array}{l}-0.162 \\
(-4.93) \\
\end{array}$ \\
\hline Obs. & 7,294 & 7,294 & 7,294 & 7,292 & 7,292 & 7,292 & 7,413 & 7,413 & 7,413 & 7,395 & 7,395 & 7,395 \\
\hline$R^{2}$ & 0.056 & 0.054 & - & 0.076 & 0.074 & - & 0.071 & 0.069 & - & 0.088 & 0.085 & - \\
\hline
\end{tabular}


Table 2 - Continued

\begin{tabular}{|c|c|c|c|c|c|c|c|c|c|c|c|c|}
\hline \multicolumn{13}{|c|}{ Panel (c) $N I_{i, t-1}<\overline{N I_{i, t-1}}$} \\
\hline \multirow[t]{2}{*}{$\beta_{0}$} & 0.004 & 0.013 & 0.024 & 0.006 & 0.016 & 0.012 & 0.006 & 0.015 & 0.013 & 0.007 & 0.016 & 0.008 \\
\hline & $(2.21)$ & $(5.65)$ & $(2.55)$ & $(2.98)$ & $(5.67)$ & $(1.10)$ & $(3.39)$ & $(6.08)$ & $(1.19)$ & $(3.49)$ & $(5.52)$ & $(0.56)$ \\
\hline \multirow{2}{*}{$\beta_{1} D_{i, t-1}$} & 0.010 & 0.007 & 0.010 & 0.012 & 0.007 & 0.026 & 0.011 & 0.008 & 0.017 & 0.014 & 0.010 & 0.027 \\
\hline & $(5.24)$ & $(3.02)$ & $(1.00)$ & $(4.88)$ & $(2.58)$ & $(2.44)$ & $(5.38)$ & $(3.28)$ & $(1.46)$ & $(6.11)$ & $(3.31)$ & $(2.09)$ \\
\hline \multirow[t]{2}{*}{$\beta_{2} N I_{i, t-1}$} & -0.086 & -0.200 & -0.356 & -0.116 & -0.242 & -0.278 & -0.108 & -0.223 & -0.232 & -0.125 & -0.247 & -0.215 \\
\hline & $(-5.75)$ & $(-9.11)$ & $(-4.17)$ & $(-6.45)$ & $(-8.98)$ & $(-2.81)$ & $(-6.95)$ & $(-9.68)$ & $(-2.20)$ & $(-7.39)$ & $(-8.83)$ & $(-1.66)$ \\
\hline \multirow[t]{2}{*}{$\beta_{3} D_{i, t-1} * N I_{i, t-1}$} & -0.186 & -0.130 & -0.161 & -0.242 & -0.166 & -0.334 & -0.224 & -0.168 & -0.267 & -0.297 & -0.221 & -0.373 \\
\hline & $(-6.79)$ & $(-4.20)$ & $(-1.39)$ & $(-7.71)$ & $(-4.67)$ & $(-2.88)$ & $(-8.61)$ & $(-5.53)$ & $(-2.08)$ & $(-10.44)$ & $(-6.20)$ & $(-2.63)$ \\
\hline Obs. & 7,505 & 7,505 & 7,505 & 7,507 & 7,507 & 7,507 & 7,625 & 7,625 & 7,625 & 7,617 & 7,617 & 7,617 \\
\hline$R^{2}$ & 0.066 & 0.063 & - & 0.091 & 0.086 & - & 0.103 & 0.098 & - & 0.128 & 0.121 & - \\
\hline
\end{tabular}

Definition of variables: $\Delta I X_{i, t}$, change in income before extraordinary items for firm $i$ from year $t-1$ to year $t$ after standard outlier detection. $\Delta N I_{i, t}$, change in net income for firm $i$ from year $t-1$ to year $t$ after standard outlier detection. $\Delta I X H_{i, t}$, change in income before extraordinary items for firm $i$ from year $t-1$ to year $t$ after outlier detection by Hadi [1994]. $\Delta N I H_{i, t}$, change in net income for firm $i$ from year $t-1$ to year $t$ after outlier detection by Hadi [1994]. All variables are standardized by total assets for firm $i$ at the end of year $t-1$.

The regressions exclude extreme $1 \%$ on each side in the standard outlier detection. The outlier detection by Hadi [1994] correspondently contains a significance level of $1 \%$.

White [1980] t-statistics in parentheses for the regressions with and without fixed effects. Windmeijer [2005] corrected z-statistics in parentheses for the regressions with the Arellano and Bond [1991] estimator. 
Table 3: Regression of changes in earnings on lagged levels in earnings for all firm-years (Enders and Granger [1998] specification)

\begin{tabular}{|c|c|c|c|c|c|c|c|c|c|c|c|c|}
\hline \multicolumn{13}{|c|}{$\Delta N I_{i, t}=\alpha_{0}+\alpha_{1} D_{i, t-1} * N I_{i, t-1}+\alpha_{2}\left(1-D_{i, t-1}\right) * N I_{i, t-1}+\epsilon_{i, t}$} \\
\hline & \multicolumn{3}{|c|}{$\Delta I X_{i, t}$} & \multicolumn{3}{|c|}{$\Delta N I_{i, t}$} & \multicolumn{3}{|c|}{$\Delta I X H_{i, t}$} & \multicolumn{3}{|c|}{$\Delta N I H_{i, t}$} \\
\hline & - & $\mathrm{Fi}$ & $\mathrm{AB}$ & - & $\mathrm{Fi}$ & $\mathrm{AB}$ & - & $\mathrm{Fi}$ & $\mathrm{AB}$ & - & $\mathrm{Fi}$ & $\mathrm{AB}$ \\
\hline & 1 & 2 & 3 & 4 & 5 & 6 & 7 & 8 & 9 & 10 & 11 & 12 \\
\hline \multicolumn{13}{|l|}{ Panel (a) $N I_{i, t-1}>0$} \\
\hline$\alpha_{0}$ & $\begin{array}{l}0.007 \\
(8.96)\end{array}$ & $\begin{array}{c}0.014 \\
(13.81)\end{array}$ & $\begin{array}{l}0.025 \\
(8.49)\end{array}$ & $\begin{array}{l}0.007 \\
(7.35)\end{array}$ & $\begin{array}{c}0.015 \\
(12.05)\end{array}$ & $\begin{array}{l}0.026 \\
(8.38)\end{array}$ & $\begin{array}{l}0.007 \\
(9.69)\end{array}$ & $\begin{array}{c}0.015 \\
(14.16)\end{array}$ & $\begin{array}{l}0.019 \\
(7.65)\end{array}$ & $\begin{array}{l}0.007 \\
(7.80)\end{array}$ & $\begin{array}{c}0.016 \\
(11.58)\end{array}$ & $\begin{array}{l}0.021 \\
(7.94)\end{array}$ \\
\hline$\alpha_{1} D_{i, t-1} * N I_{i, t-1}$ & $\begin{array}{c}-0.112 \\
(-13.33)\end{array}$ & $\begin{array}{c}-0.215 \\
(-15.76)\end{array}$ & $\begin{array}{c}-0.354 \\
(-11.27)\end{array}$ & $\begin{array}{c}-0.123 \\
(-12.33)\end{array}$ & $\begin{array}{c}-0.236 \\
(-14.44)\end{array}$ & $\begin{array}{c}-0.390 \\
(-11.65)\end{array}$ & $\begin{array}{c}-0.120 \\
(-14.23)\end{array}$ & $\begin{array}{c}-0.227 \\
(-16.30)\end{array}$ & $\begin{array}{l}-0.276 \\
(-9.34)\end{array}$ & $\begin{array}{c}-0.130 \\
(-13.26)\end{array}$ & $\begin{array}{c}-0.242 \\
(-14.07)\end{array}$ & $\begin{array}{c}-0.316 \\
(-10.01)\end{array}$ \\
\hline$\alpha_{2}\left(1-D_{i, t-1}\right) * N I_{i, t-1}$ & $\begin{array}{l}-0.620 \\
(-8.51) \\
\end{array}$ & $\begin{array}{l}-0.591 \\
(-8.06) \\
\end{array}$ & $\begin{array}{l}-0.836 \\
(-7.29) \\
\end{array}$ & $\begin{array}{c}-0.738 \\
(-10.79)\end{array}$ & $\begin{array}{l}-0.697 \\
(-9.78) \\
\end{array}$ & $\begin{array}{c}-0.950 \\
(-10.81)\end{array}$ & $\begin{array}{c}-0.681 \\
(-13.20)\end{array}$ & $\begin{array}{c}-0.658 \\
(-11.78)\end{array}$ & $\begin{array}{l}-0.832 \\
(-9.28) \\
\end{array}$ & $\begin{array}{c}-0.767 \\
(-15.48)\end{array}$ & $\begin{array}{c}-0.744 \\
(-12.09)\end{array}$ & $\begin{array}{c}-0.876 \\
(-11.42) \\
\end{array}$ \\
\hline Obs. & 7,505 & 7,505 & 7,505 & 7,507 & 7,507 & 7,507 & 7,625 & 7,625 & 7,625 & 7,617 & 7,617 & 7,617 \\
\hline$R^{2}$ & 0.070 & 0.066 & - & 0.101 & 0.095 & - & 0.114 & 0.107 & - & 0.141 & 0.133 & - \\
\hline \multicolumn{13}{|l|}{ Panel (b) $\Delta N I_{i, t-1}>0$} \\
\hline$\alpha_{0}$ & $\begin{array}{c}0.009 \\
(12.52)\end{array}$ & $\begin{array}{c}0.017 \\
(16.36)\end{array}$ & $\begin{array}{l}0.028 \\
(8.74)\end{array}$ & $\begin{array}{c}0.012 \\
(12.69)\end{array}$ & $\begin{array}{c}0.021 \\
(17.09)\end{array}$ & $\begin{array}{c}0.032 \\
(11.35)\end{array}$ & $\begin{array}{c}0.011 \\
(14.20)\end{array}$ & $\begin{array}{c}0.019 \\
(14.16)\end{array}$ & $\begin{array}{c}0.025 \\
(11.52)\end{array}$ & $\begin{array}{c}0.013 \\
(13.98)\end{array}$ & $\begin{array}{c}0.022 \\
(17.97)\end{array}$ & $\begin{array}{c}0.028 \\
(11.51)\end{array}$ \\
\hline$\alpha_{1} D N I_{i, t-1} * N I_{i, t-1}$ & $\begin{array}{c}-0.126 \\
(-14.29)\end{array}$ & $\begin{array}{c}-0.226 \\
(-17.16)\end{array}$ & $\begin{array}{l}-0.356 \\
(-9.26)\end{array}$ & $\begin{array}{c}-0.170 \\
(-15.55)\end{array}$ & $\begin{array}{c}-0.280 \\
(-17.41)\end{array}$ & $\begin{array}{c}-0.423 \\
(-12.82)\end{array}$ & $\begin{array}{c}-0.137 \\
(-16.49)\end{array}$ & $\begin{array}{l}-0.240 \\
(-19.53)\end{array}$ & $\begin{array}{c}-0.312 \\
(-12.05)\end{array}$ & $\begin{array}{c}-0.180 \\
(-17.41)\end{array}$ & $\begin{array}{c}-0.292 \\
(-18.54)\end{array}$ & $\begin{array}{l}-0.373 \\
(-12.08)\end{array}$ \\
\hline$\alpha_{2}\left(1-D N I_{i, t-1}\right) * N I_{i, t-1}$ & $\begin{array}{c}-0.151 \\
(-15.41)\end{array}$ & $\begin{array}{c}-0.264 \\
(-15.90)\end{array}$ & $\begin{array}{c}-0.417 \\
(-11.22)\end{array}$ & $\begin{array}{c}-0.191 \\
(-15.74)\end{array}$ & $\begin{array}{c}-0.315 \\
(-17.22)\end{array}$ & $\begin{array}{c}-0.484 \\
(-15.25)\end{array}$ & $\begin{array}{c}-0.164 \\
(-16.69)\end{array}$ & $\begin{array}{c}-0.279 \\
(-18.36)\end{array}$ & $\begin{array}{c}-0.379 \\
(-14.25)\end{array}$ & $\begin{array}{c}-0.201 \\
(-16.95)\end{array}$ & $\begin{array}{c}-0.326 \\
(-17.83)\end{array}$ & $\begin{array}{c}-0.431 \\
(-13.31) \\
\end{array}$ \\
\hline Obs. & 7,294 & 7,294 & 7,294 & 7,292 & 7,292 & 7,292 & 7,413 & 7,413 & 7,413 & 7,395 & 7,395 & 7,395 \\
\hline$R^{2}$ & 0.052 & 0.052 & - & 0.069 & 0.069 & - & 0.066 & 0.066 & - & 0.080 & 0.080 & - \\
\hline
\end{tabular}

Definition of variables: $\Delta I X_{i, t}$, change in income before extraordinary items for firm $i$ from year $t-1$ to year $t$ after standard outlier detection. $\Delta N I_{i, t}$, change in net income for firm $i$ from year $t-1$ to year $t$ after standard outlier detection. $\Delta I X H_{i, t}$, change in income before extraordinary items for firm $i$ from year $t-1$ to year $t$ after outlier detection by Hadi [1994]. $\Delta N I H_{i, t}$, change in net income for firm $i$ from year $t-1$ to year $t$ after outlier detection by Hadi [1994]. All variables are standardized by total assets for firm $i$ at the end of year $t-1$.

The regressions exclude extreme $1 \%$ on each side in the standard outlier detection. The outlier detection by Hadi [1994] correspondently contains a significance level of $1 \%$.

White [1980] t-statistics in parentheses for the regressions with and without fixed effects. Windmeijer [2005] corrected z-statistics in parentheses for the regressions with the Arellano and Bond [1991] estimator. 
Negative changes in income are followed by relatively large positive changes in income, the main result in the literature.

In Table 2, we report the results from our modified regression of the changes on the lagged levels of income (see equation 4). We estimate this regression with three alternative definitions of the dummy variable. In panel (a), $D_{i, t-1}$ is equal to one, when the lagged changes are negative (as in Basu [1997] and Ball and Shivakumar [2005]), in panel (b) it is equal to one, when the lagged levels are negative and in panel (c) it is equal to one, when income is below the average. Focusing on the coefficient $\beta_{3}$, we, again, confirm the main findings of Basu [1997] and Ball and Shivakumar [2005] on the asymmetric timeliness of earnings, also when using our alternative approach: In all regressions, the coefficients are negative and statistically significant. The magnitude of the coefficients indicates that about $20-80 \%$ of the shocks are absorbed one year after they occur, again a finding that is - although quantitatively somewhat larger - broadly consistent with other studies. Our alternative specification does therefore not appear to change the major conclusions from the literature on conservatism when applied to a common data set. This is also the case, when using the Enders and Granger [1998] TAR approach in its original form, as shown in Table 3. Again, we find that the mean reversion after negative changes/levels is faster, when these changes/levels are negative.

There are, however, some differences in the other coefficients of the regression. For instance in Table 2, we find that all $\beta_{2}$ coefficients are negative and statistically significant. This implies that the positive shocks in accounting income are also transitory although relatively less transitory than negative shocks. This finding is consistent with the basic assumption in Basu [1997] and Ball et al. [2009] who argue that income in general is transitory.

There may furthermore be larger differences when the sample gets smaller, as it is the case in many other data sets. Table 7 in the Appendix shows that the t-statistics, respectively z-statistics, of the coefficients are substantially reduced, when the sample period is shortened to the period of 1980 onwards. While the coefficients on negative changes in the Basu [1997] regression remain significant at the 5\%-level, all but one of the coefficients on $\alpha_{2}$ are insignificant in the shorter sample.

In a final robustness test, we also implement the test, including both the lagged levels and lagged changes, similar to an augmented Dickey-Fuller test ${ }^{8}$. In this regression the interpretation on the lagged levels does not change. The lagged differences are serving as a further control variable to correct for possibly remaining autocorrelation in the residuals. Table 4 shows that the results remain qualitatively unchanged with respect to regression 4 .

\footnotetext{
${ }^{8}$ We also tried higher lag structures which remain insignificant, however.
} 
Table 4: Regression of the augmented Dickey-Fuller Test for all firm-years

\begin{tabular}{|c|c|c|c|c|c|c|c|c|c|c|c|c|}
\hline & \multicolumn{2}{|c|}{$\Delta I X_{i, t}$} & \multicolumn{2}{|c|}{$\Delta N I_{i, t}$} & \multicolumn{2}{|c|}{$\Delta I X H_{i, t}$} & \multicolumn{2}{|c|}{$\Delta N I H_{i, t}$} & \multirow[b]{2}{*}{$\mathrm{AB}$} & \multirow[b]{2}{*}{ - } & \multirow[b]{2}{*}{$\mathrm{Fi}$} & \multirow[b]{2}{*}{$\mathrm{AB}$} \\
\hline & - & $\mathrm{Fi}$ & $\mathrm{AB}$ & - & $\mathrm{Fi}$ & $\mathrm{AB}$ & - & $\mathrm{Fi}$ & & & & \\
\hline & 1 & 2 & 3 & 4 & 5 & 6 & 7 & 8 & 9 & 10 & 11 & 12 \\
\hline$\beta_{0}$ & $\begin{array}{l}0.006 \\
(7.57)\end{array}$ & $\begin{array}{c}0.014 \\
(12.07)\end{array}$ & $\begin{array}{l}0.023 \\
(4.72)\end{array}$ & $\begin{array}{l}0.005 \\
(5.64)\end{array}$ & $\begin{array}{c}0.014 \\
(10.51)\end{array}$ & $\begin{array}{l}0.022 \\
(5.48)\end{array}$ & $\begin{array}{l}0.006 \\
(8.16)\end{array}$ & $\begin{array}{c}0.014 \\
(12.95)\end{array}$ & $\begin{array}{l}0.019 \\
(3.87)\end{array}$ & $\begin{array}{l}0.005 \\
(5.74)\end{array}$ & $\begin{array}{c}0.013 \\
(10.45)\end{array}$ & $\begin{array}{l}0.017 \\
(2.71)\end{array}$ \\
\hline$\beta_{1} D_{i, t-1}$ & $\begin{array}{l}0.007 \\
(1.76)\end{array}$ & $\begin{array}{l}0.005 \\
(1.38)\end{array}$ & $\begin{array}{l}0.006 \\
(0.95)\end{array}$ & $\begin{array}{l}0.005 \\
(1.20)\end{array}$ & $\begin{array}{l}0.004 \\
(0.94)\end{array}$ & $\begin{array}{l}0.011 \\
(1.53)\end{array}$ & $\begin{array}{l}0.008 \\
(2.46)\end{array}$ & $\begin{array}{l}0.007 \\
(1.99)\end{array}$ & $\begin{array}{l}0.006 \\
(0.99)\end{array}$ & $\begin{array}{l}0.005 \\
(1.27)\end{array}$ & $\begin{array}{l}0.004 \\
(0.80)\end{array}$ & $\begin{array}{c}0.004 \\
(0.56)\end{array}$ \\
\hline$\beta_{2} N I_{i, t-1}$ & $\begin{array}{c}-0.094 \\
(-11.34)\end{array}$ & $\begin{array}{c}-0.200 \\
(-13.60)\end{array}$ & $\begin{array}{l}-0.338 \\
(-5.48)\end{array}$ & $\begin{array}{c}-0.098 \\
(-10.06)\end{array}$ & $\begin{array}{c}-0.210 \\
(-12.71)\end{array}$ & $\begin{array}{l}-0.327 \\
(-7.04)\end{array}$ & $\begin{array}{c}-0.098 \\
(-12.28)\end{array}$ & $\begin{array}{c}-0.205 \\
(-14.60)\end{array}$ & $\begin{array}{l}-0.281 \\
(-4.29)\end{array}$ & $\begin{array}{c}-0.100 \\
(-10.68)\end{array}$ & $\begin{array}{c}-0.208 \\
(-12.92)\end{array}$ & $\begin{array}{l}-0.261 \\
(-3.30\end{array}$ \\
\hline$\beta_{3} D_{i, t-1} * N I_{i, t-1}$ & $\begin{array}{l}-0.356 \\
(-3.12)\end{array}$ & $\begin{array}{l}-0.287 \\
(-2.48)\end{array}$ & $\begin{array}{l}-0.530 \\
(-2.67)\end{array}$ & $\begin{array}{l}-0.481 \\
(-4.26)\end{array}$ & $\begin{array}{l}-0.392 \\
(-4.09)\end{array}$ & $\begin{array}{l}-0.498 \\
(-3.68)\end{array}$ & $\begin{array}{l}-0.328 \\
(-4.06)\end{array}$ & $\begin{array}{l}-0.257 \\
(-3.06)\end{array}$ & $\begin{array}{l}-0.526 \\
(-2.94)\end{array}$ & $\begin{array}{l}-0.496 \\
(-6.05)\end{array}$ & $\begin{array}{l}-0.423 \\
(-4.79)\end{array}$ & $\begin{array}{l}-0.619 \\
(-4.37)\end{array}$ \\
\hline$\beta_{4} \Delta N I_{i, t-1}$ & $\begin{array}{l}-0.042 \\
(-2.62)\end{array}$ & $\begin{array}{l}-0.003 \\
(-0.15)\end{array}$ & $\begin{array}{l}0.053 \\
(3.73)\end{array}$ & $\begin{array}{l}-0.099 \\
(-5.61)\end{array}$ & $\begin{array}{l}-0.062 \\
(-3.28)\end{array}$ & $\begin{array}{l}0.016 \\
(0.87)\end{array}$ & $\begin{array}{l}-0.052 \\
(-3.45)\end{array}$ & $\begin{array}{l}-0.015 \\
(-0.86)\end{array}$ & $\begin{array}{l}0.058 \\
(3.32)\end{array}$ & $\begin{array}{l}-0.109 \\
(-6.58)\end{array}$ & $\begin{array}{l}-0.072 \\
(-3.81)\end{array}$ & $\begin{array}{l}-0.006 \\
(-0.26)\end{array}$ \\
\hline Obs. & 7,294 & 7,294 & 7,294 & 7,292 & 7,292 & 7,292 & 7,413 & 7,413 & 7,413 & 7,395 & 7,395 & 7,395 \\
\hline$R^{2}$ & 0.067 & 0.061 & - & 0.104 & 0.095 & - & 0.088 & 0.081 & 0.124 & 0.115 & - & \\
\hline
\end{tabular}

Definition of variables: $\Delta I X_{i, t}$, change in income before extraordinary items for firm $i$ from year $t-1$ to year $t$ after standard outlier detection. $\Delta N I_{i, t}$, change in net income for firm $i$ from year $t-1$ to year $t$ after standard outlier detection. $\Delta I X H_{i, t}$, change in income before extraordinary items for firm $i$ from year $t-1$ to year $t$ after outlier detection by Hadi [1994]. $\Delta N I H_{i, t}$, change in net income for firm $i$ from year $t-1$ to year $t$ after outlier detection by Hadi [1994]. $D_{i, t-1}=1$ if $N I_{i, t-1}<0 ;=0$ otherwise. All variables are standardized by total assets for firm $i$ at the end of year $t-1$.

The regressions exclude extreme $1 \%$ on each side in the standard outlier detection. The outlier detection by Hadi [1994] correspondently contains a significance level of $1 \%$.

White [1980] t-statistics in parentheses for the regressions with and without fixed effects. Windmeijer [2005] corrected z-statistics in parentheses for the regressions with the Arellano and Bond [1991] estimator. 
Table 5: Results of the Monte Carlo Simulation

\begin{tabular}{ccccc}
\hline (a) $\Delta x_{t}=\beta * \Delta x_{t-1}+\epsilon_{t}$, & & & \\
\hline $\begin{array}{c}\text { Simulated } \\
\text { coefficient (in levels) }\end{array}$ & $\begin{array}{c}\text { Estimated } \\
\text { coefficient }\end{array}$ & t-statistics & $R^{2}$ & $\begin{array}{c}\text { Annual } \\
\text { persistence }\end{array}$ \\
\hline$\alpha=0$ & -0.4846 & -55.3861 & 0.2348 & 0.5154 \\
$\alpha=0.05$ & -0.4594 & -51.7108 & 0.2110 & 0.5406 \\
$\alpha=0.31$ & -0.3291 & -34.8468 & 0.1083 & 0.6709 \\
$\alpha=0.95$ & -0.0139 & -1.3922 & 0.0002 & 0.9861 \\
$\alpha=1$ & 0.0085 & 0.8489 & 0.0000 & 1.0085 \\
\hline (b) $\Delta x_{t}=\beta * x_{t-1}+\epsilon_{t}$ & & & \\
\hline$\alpha=0$ & -0.9914 & -99.1411 & 0.4957 & 0.0086 \\
$\alpha=0.05$ & -0.9426 & -94.4103 & 0.4713 & 0.0574 \\
$\alpha=0.31$ & -0.6888 & -72.4676 & 0.3444 & 0.3112 \\
$\alpha=0.95$ & -0.0553 & -16.8203 & 0.0275 & 0.9447 \\
$\alpha=1$ & 0.0000 & -0.0666 & -0.0001 & 1.0000 \\
\hline
\end{tabular}

\section{A Monte Carlo Simulation}

The empirical exercise above largely confirms the findings of the literature on conservatism, despite the conceptual advantages of the unit root-type test setup discussed in section 2 . In this section, we aim to explain our results, by using a Monte Carlo Simulation. The key advantage of a simulated data set is that we know in advance the true data generating process and we can use the two regression specifications to test which one is able to identify this process more precisely.

We start by drawing 10,000 observations from a normal distribution. We then simulate an $\operatorname{AR}(1)$ process, $x_{t}=\alpha x_{t-1}+\epsilon_{t}$, using different values for $\alpha$. We finally estimate two regressions to test for persistence in this new data set. One, where we regress the changes of the data on the lagged changes, $\Delta x_{t}=\beta \Delta x_{t-1}+\epsilon_{t}$, and one where we regress the changes on the lagged levels, $\Delta x_{t}=\beta x_{t-1}+\epsilon_{t}$. In order to keep the simulation simple we do not include an intercept, and abstract from the issue of asymmetry between positive and negative shocks.

The results of the regressions are displayed in Table 5 . We find that for most $0<\alpha<1$, the negative and significant values of $\beta$ indicate that both approaches pick up the mean reversion that is truly present in the data. This explains why conservatism, that very likely exists in most real world data sets, is identified by the Basu [1997] and the subsequent literature, as well as in our alternative regression setup. However, we also find that the unit root test identifies the magnitude of the mean reversion of shocks more precisely. For instance, when the degree of persistence of the shocks in levels is 0.31 (the benchmark for negative shocks in Basu [1997], who reports a mean reversion of -0.69 in the first year), the unit root test identifies a coefficient of -0.6863 - which corresponds to an annual persistence level of 0.3137. In the regression of changes on lagged changes, we find only a coefficient of -0.3291 , which corresponds to an annual persistence level of 0.6709 (when only considering the first year effect), substantially less mean reversion than reported in Basu [1997]. The negative autocorrelation in the changes 
of the $\mathrm{AR}(1)$ process, thus appears to be only a somewhat noisy signal of the true mean reversion in the data set.

In a next step, we vary the magnitude of the actual mean reversion in the simulated time series. Table 5 shows that in the case of $\alpha=0$, a perfect mean reversion in levels, both regressions indicate a highly significant negative coefficient. However, the regression of changes on lagged changes again underestimates the true degree of mean reversion, a result that we find for all levels of mean reversion in our simulated data set. When the persistence levels are relatively high, for instance for an $\alpha=0.95$, there is also a qualitative difference in the results. While the unit root test still identifies the mean reversion, with a t-statistic of -16.82 , the negative coefficient in the regression of changes on lagged changes has become insignificant with a t-statistic of only -1.39. This simulation finding for high persistence levels helps to explain why the literature typically reports persistence for positive shocks (a regression coefficient equal to zero), while we still find some mean reversion, although very slowly and significantly different from that of negative shocks.

\section{Related literature}

The correct specification for accounting income has generated a long debate in the field of empirical accounting research. In an early paper, Ball and Watts [1972] show that the first differences of income are uncorrelated over time which implies that income changes are persistent. An asymmetry between positive an negative shocks in the persistence levels has been first pointed out by Brooks and Buckmaster [1976]. Basu [1997] uses this stylized fact to reverse the argument and interprets a negative correlation of successive income changes, after a negative shock, as an indicator for transitory elements in the time series, and gives an interpretation to this phenomenon in the context of conservatism. Other papers that recently followed this approach are Ball et al. [2003], Ball and Shivakumar [2005] and Ball et al. [2009].

Some papers tried to challenge the original Ball and Watts [1972] finding. For instance Ali and Zarowin [1992] have pointed out that the change in net income is not a good predictor of shocks, when net income is not a random walk. Our argument is related to Ali and Zarowin [1992], in that we also argue that the error term - and the response of the variable to a shock over time - rather than the lagged changes should be taken as 'news'.

A similar perspective is also taken in some papers that focus on the forecasting properties of net income. Albrecht et al. [1977], Finger [1994] and Gil-Alana and Peláez [2008] treat income as a stochastic ARIMA process. While Albrecht et al. [1977] were not able to reject the random walk hypothesis, Finger [1994] finds some scope for forecastability, using the long run cointegration properties. Finger [1994] is also quoted in the Basu [1997] and Ball and Shivakumar [2005] literature on the asymmetric timeliness, as Finger [1994] found that it is optimal to difference the data, due to the unit roots in the majority of cases. Note, however, 
that this was done purely for forecasting purposes. It does not follow from the Finger [1994] paper that the differencing of the data is also appropriate for conducting tests of persistence.

Fama and French [2000] also perform a non-linear estimation, very similar to the Basu [1997] setup, taking profitability as well as earnings as their main variable of interest. In this paper, the main focus is on the forecasting properties of the changes in profitability and changes in earnings. They do not have a prior, however, on the impulse response pattern, as given in Figure 1b of Basu [1997]. In fact, an oscillating pattern in the changes of earnings can be explained in their model, as a result of an oscillating pattern in the changes of profits. While the oscillation does not constitute a problem from a forecasting perspective, it seems more difficult to be reconciled with the interpretation of conservatism driving the negative coefficient.

Hayn [1995], as well as Basu [1995] have taken a similar approach to Basu [1997], regressing levels on lagged levels, rather than changes on lagged changes. More recently, papers by Basu [1997], Ball and Shivakumar [2005] and Ball et al. [2009] have argued that changes in income reflect news more appropriately than the levels of income. Our setup differs from both of the two approaches, as they focus primarily on the systematic link between values in period $t$ and period $t-1$ which is captured directly by the coefficient $\alpha_{1}$, while we focus on the question of how an unexpected change - a 'shock' in net income that is captured by the error term $\epsilon_{i, t}$ - will be incorporated in future income statements. The asymmetry in the response to this shock would be an alternative way to measure conservatism ${ }^{9}$.

A critique of the levels specification in Basu [1997], Ball and Shivakumar [2005] and Ball et al. [2009] is the survivorship bias and it cannot easily be dismissed, for both the levels specification, and for our own regression. Do firms with negative income in levels disappear from the data set? In our note and the empirical exercise, we try to limit this problem, taking long time series data, and focus in a sample of firms that remain in the sample for the entire period (from the foundation of the index up to today). There still remain 539 firm-years with negative income, out of a total of 7,888 firm-years that can be analyzed. We further address this issue by using different definitions of the dummy variable for lagged values, including lagged changes where we do not condition on actual losses. The aim of this note is to focus on the technical aspects of the estimation. In order to generalize from the results reported in this paper, however, it would be necessary to broaden the data base and to analyze different types of firms in future research.

Finally, the discussion on the persistence of income shocks has played an important role in

\footnotetext{
${ }^{9}$ For a pure $\mathrm{AR}(1)$ process, our specification is in fact equivalent to a regression of levels on lagged levels in earnings, while a regression of changes on lagged changes is not. Starting off with a setup in levels, and subtracting $N I_{i, t-1}$ on both sides leads to the unit root-type test specification. When differencing both sides of the equation, one subtracts $N I_{i, t-1}$ on the left and $N I_{i, t-2}$ on the right side - which is not an equivalent transformation. The interpretation of the coefficient therefore changes as well. Our coefficient $\beta_{1}$ is equivalent to the coefficient $\alpha_{1}=0$ in a levels-regression, -1 . Most empirical studies, however, have found it more convenient to implement the Dickey-Fuller specification, as it implies a simple test on whether a coefficient is zero (rather than one) and it can be easily extended for higher lag orders.
} 
economics, when looking at aggregate income, such as gross domestic product. Motivated by the permanent income hypothesis, several researchers have aimed to test, whether aggregate national income is persistent or transitory. In this debate, Nelson and Plosser [1982], Campbell and Mankiw [1987] and Cheung and Chinn [1997], among others, have taken a unit root type specification to address this issue. 


\section{Appendix}

Table 6: Regression of changes in earnings on lagged changes in earnings for all firm-years (1980-2008 (Basu [1997] specification)

\begin{tabular}{|c|c|c|c|c|c|c|c|c|c|c|c|c|}
\hline \multicolumn{13}{|c|}{$\Delta N I_{i, t}=\alpha_{0}+\alpha_{1} D_{i, t-1}+\alpha_{2} \Delta N I_{i, t-1}+\alpha_{3} D_{i, t-1} * \Delta N I_{i, t-1}+\epsilon_{i, t}$} \\
\hline & \multicolumn{3}{|c|}{$\Delta I X_{i, t}$} & \multicolumn{3}{|c|}{$\Delta N I_{i, t}$} & \multicolumn{3}{|c|}{$\Delta I X H_{i, t}$} & \multicolumn{3}{|c|}{$\Delta N I H_{i, t}$} \\
\hline & - & $\mathrm{Fi}$ & $\mathrm{AB}$ & - & $\mathrm{Fi}$ & $\mathrm{AB}$ & - & Fi & $\mathrm{AB}$ & - & $\mathrm{Fi}$ & $\mathrm{AB}$ \\
\hline & 1 & 2 & 3 & 4 & 5 & 6 & 7 & 8 & 9 & 10 & 11 & 12 \\
\hline \multirow[t]{2}{*}{$\alpha_{0}$} & -0.005 & -0.006 & -0.007 & -0.006 & -0.007 & -0.010 & -0.005 & -0.006 & -0.006 & -0.006 & -0.008 & -0.008 \\
\hline & $(-4.97)$ & $(-6.31)$ & $(-2.64)$ & $(-4.95)$ & $(-5.86)$ & $(-3.86)$ & $(-5.18)$ & $(-6.30)$ & $(-2.74)$ & $(-5.67)$ & $(-6.49)$ & $(-3.73)$ \\
\hline \multirow[t]{2}{*}{$\alpha_{1} D_{i, t-1}$} & -0.003 & -0.003 & 0.001 & -0.006 & -0.006 & 0.003 & -0.004 & -0.004 & -0.001 & -0.006 & -0.006 & -0.001 \\
\hline & $(-2.20)$ & $(-2.03)$ & $(0.33)$ & $(-3.29)$ & $(-3.22)$ & $(1.04)$ & $(-2.88)$ & $(-2.50)$ & $(-0.58)$ & $(-3.24)$ & $(-3.22)$ & $(-0.24)$ \\
\hline \multirow[t]{2}{*}{$\alpha_{2} \Delta N I_{i, t-1}$} & 0.022 & 0.061 & 0.125 & -0.074 & -0.035 & 0.066 & 0.009 & 0.044 & 0.068 & -0.069 & -0.028 & -0.028 \\
\hline & $(0.50)$ & $(1.50)$ & $(1.24)$ & $(-1.66)$ & $(-0.76)$ & $(0.76)$ & $(0.24)$ & (1.19) & $(0.93)$ & $(-1.82)$ & $(-0.67)$ & $(-0.46)$ \\
\hline \multirow{2}{*}{$\alpha_{3} D_{i, t-1} * \Delta N I_{i, t-1}$} & -0.351 & -0.445 & -0.409 & -0.408 & -0.507 & -0.476 & -0.397 & -0.478 & -0.383 & -0.428 & -0.527 & -0.393 \\
\hline & $(-5.66)$ & $(-6.36)$ & $(-2.29)$ & $(-6.51)$ & $(-6.41)$ & $(-3.12)$ & $(-7.35)$ & $(-7.78)$ & $(-2.52)$ & $(-7.48)$ & $(-6.87)$ & $(-3.57)$ \\
\hline Obs. & 3,733 & 3,733 & 3,733 & 3,710 & 3,710 & 3,710 & 3,780 & 3,780 & 3,780 & 3,756 & 3,756 & 3,756 \\
\hline$R^{2}$ & 0.032 & 0.031 & - & 0.073 & 0.073 & - & 0.045 & 0.045 & - & 0.081 & 0.081 & - \\
\hline
\end{tabular}

Definition of variables: $\Delta I X_{i, t}$, change in income before extraordinary items for firm $i$ from year $t-1$ to year $t$ after standard outlier detection. $\Delta N I_{i, t}$, change in net income for firm $i$ from year $t-1$ to year $t$ after standard outlier detection. $\Delta I X H_{i, t}$, change in income before extraordinary items for firm $i$ from year $t-1$ to year $t$ after outlier detection by Hadi [1994]. $\Delta N I H_{i, t}$, change in net income for firm $i$ from year $t-1$ to year $t$ after outlier detection by Hadi [1994]. $D_{i, t-1}=1$ if $\Delta N I_{i, t-1}<0 ;=0$ otherwise. All variables are standardized by total assets for firm $i$ at the end of year $t-1$.

The regressions exclude extreme $1 \%$ on each side in the standard outlier detection. The outlier detection by Hadi [1994] correspondently contains a significance level of $1 \%$.

White [1980] t-statistics in parentheses for the regressions with and without fixed effects. Windmeijer [2005] corrected z-statistics in parentheses for the regressions with the Arellano and Bond [1991] estimator. 
Table \%: Regression of change in earnings on lagged levels of earnings for all firm-years (1980-2008)

\begin{tabular}{|c|c|c|c|c|c|c|c|c|c|c|c|c|}
\hline \multicolumn{13}{|c|}{$\Delta N I_{i, t}=\beta_{0}+\beta_{1} D_{i, t-1}+\beta_{2} D_{i, t-1}+\beta_{3} D_{i, t-1} * N I_{i, t-1}+\epsilon_{i, t}$} \\
\hline & - & $\mathrm{Fi}$ & $\mathrm{AB}$ & - & $\mathrm{Fi}$ & $\mathrm{AB}$ & - & $\mathrm{Fi}$ & $\mathrm{AB}$ & - & $\mathrm{Fi}$ & $\mathrm{AB}$ \\
\hline & 1 & 2 & 3 & 4 & 5 & 6 & 7 & 8 & 9 & 10 & 11 & 12 \\
\hline$\beta_{0}$ & $\begin{array}{l}0.004 \\
(3.72)\end{array}$ & $\begin{array}{c}0.016 \\
(12.44)\end{array}$ & $\begin{array}{l}0.016 \\
(4.11)\end{array}$ & $\begin{array}{l}0.004 \\
(2.77)\end{array}$ & $\begin{array}{c}0.018 \\
(10.34)\end{array}$ & $\begin{array}{l}0.019 \\
(4.19)\end{array}$ & $\begin{array}{l}0.005 \\
(4.22)\end{array}$ & $\begin{array}{c}0.017 \\
(13.14)\end{array}$ & $\begin{array}{l}0.012 \\
(3.64)\end{array}$ & $\begin{array}{l}0.004 \\
(2.73)\end{array}$ & $\begin{array}{l}0.018 \\
(9.54)\end{array}$ & $\begin{array}{l}0.014 \\
(3.53)\end{array}$ \\
\hline$\beta_{1} D_{i, t-1}$ & $\begin{array}{l}0.007 \\
(1.68)\end{array}$ & $\begin{array}{l}0.005 \\
(1.37)\end{array}$ & $\begin{array}{l}0.008 \\
(2.07)\end{array}$ & $\begin{array}{l}0.006 \\
(1.24)\end{array}$ & $\begin{array}{l}0.004 \\
(0.90)\end{array}$ & $\begin{array}{l}0.013 \\
(2.41)\end{array}$ & $\begin{array}{l}0.005 \\
(1.46)\end{array}$ & $\begin{array}{l}0.004 \\
(1.26)\end{array}$ & $\begin{array}{l}0.009 \\
(2.35)\end{array}$ & $\begin{array}{l}0.007 \\
(1.49)\end{array}$ & $\begin{array}{l}0.004 \\
(0.82)\end{array}$ & $\begin{array}{c}0.009 \\
(1.64)\end{array}$ \\
\hline$\beta_{2} N I_{i, t-1}$ & $\begin{array}{l}-0.120 \\
(-9.22)\end{array}$ & $\begin{array}{c}-0.303 \\
(-16.06)\end{array}$ & $\begin{array}{l}-0.292 \\
(-6.52)\end{array}$ & $\begin{array}{l}-0.143 \\
(-8.92)\end{array}$ & $\begin{array}{c}-0.354 \\
(-14.79)\end{array}$ & $\begin{array}{l}-0.365 \\
(-6.94)\end{array}$ & $\begin{array}{l}-0.129 \\
(-9.87)\end{array}$ & $\begin{array}{c}-0.320 \\
(-17.02)\end{array}$ & $\begin{array}{l}-0.246 \\
(-5.81)\end{array}$ & $\begin{array}{l}-0.148 \\
(-9.22)\end{array}$ & $\begin{array}{c}-0.357 \\
(-13.65)\end{array}$ & $\begin{array}{l}-0.301 \\
(-6.00)\end{array}$ \\
\hline$\beta_{3} D_{i, t-1} * N I_{i, t-1}$ & $\begin{array}{l}-0.367 \\
(-3.21) \\
\end{array}$ & $\begin{array}{l}-0.222 \\
(-1.98) \\
\end{array}$ & $\begin{array}{l}-0.470 \\
(-3.60) \\
\end{array}$ & $\begin{array}{l}-0.498 \\
(-4.59) \\
\end{array}$ & $\begin{array}{l}-0.305 \\
(-3.25) \\
\end{array}$ & $\begin{array}{l}-0.427 \\
(-3.46) \\
\end{array}$ & $\begin{array}{l}-0.481 \\
(-6.54) \\
\end{array}$ & $\begin{array}{l}-0.306 \\
(-4.21) \\
\end{array}$ & $\begin{array}{l}-0.498 \\
(-4.33) \\
\end{array}$ & $\begin{array}{l}-0.550 \\
(-7.24) \\
\end{array}$ & $\begin{array}{l}-0.373 \\
(-4.05) \\
\end{array}$ & $\begin{array}{l}-0.504 \\
(-4.03) \\
\end{array}$ \\
\hline Obs. & 3,764 & 3,764 & 3,764 & 3,751 & 3,751 & 3,751 & 3,830 & 3,830 & 3,830 & 3,820 & 3,820 & 3,820 \\
\hline$R^{2}$ & 0.086 & 0.079 & - & 0.123 & 0.112 & - & 0.141 & 0.128 & - & 0.170 & 0.157 & - \\
\hline
\end{tabular}

Definition of variables: $\Delta I X_{i, t}$, change in income before extraordinary items for firm $i$ from year $t-1$ to year $t$ after standard outlier detection. $\Delta N I_{i, t}$, change in net income for firm $i$ from year $t-1$ to year $t$ after standard outlier detection. $\Delta I X H_{i, t}$, change in income before extraordinary items for firm $i$ from year $t-1$ to year $t$ after outlier detection by Hadi [1994]. $\Delta N I H_{i, t}$, change in net income for firm $i$ from year $t-1$ to year $t$ after outlier detection by Hadi [1994]. $D_{i, t-1}=1$ if $N I_{i, t-1}<0 ;=0$ otherwise. All variables are standardized by total assets for firm $i$ at the end of year $t-1$.

The regressions exclude extreme $1 \%$ on each side in the standard outlier detection. The outlier detection by Hadi [1994] correspondently contains a significance level of $1 \%$.

White [1980] t-statistics in parentheses for the regressions with and without fixed effects. Windmeijer [2005] corrected z-statistics in parentheses for the regressions with the Arellano and Bond [1991] estimator. 


\section{References}

ALBRECHT, W. S., LOOKABILL, L. L., and MCKEOWN, J. C. 'The Time Series Properties of Annual Earnings.' Journal of Accounting Research 15 (1977): 226-244.

ALI, A., and ZAROWIN, P. 'Permanent Versus Transitory Components of Annual Earnings and Estimation Error in Earnings Response Coefficients.' Journal of Accounting and Economics 15 (1992): 249-264.

ARELlano, M., and BOND, S. 'Some Tests of Specification for Panel Data: Monte Carlo Evidence and an Application to Employment Equations.' Review of Economic Studies 58 (1991): 277-297.

BALL, R., KOTHARI, S., and NIKOLAEV, V. 'Econometrics of the Basu Asymmetric Timeliness Coefficient and Accounting Conservatism,' Unpublished paper, The University of Chicago Booth School of Business, 2009. Available at http://ssrn.com/abstract $=999710$.

BALL, R., ROBIN, A., and WU, J. S. 'Incentives Versus Standards: Properties of Accounting Income in Four East Asian countries.' Journal of Accounting and Economics 36 (2003): 235-270.

BALL, R., and SHIVAKUMAR, L. 'Earnings Quality in UK Private Firms: Comparative Loss Recognition Timeliness.' Journal of Accounting and Economics 39 (2005): 83-128.

BALL, R., and WATTS, R. 'Some Time Series Properties of Accounting Income.' The Journal of Finance 27 (1972): 663-681.

BASU, S. Conservatism and the Asymmetric Timeliness of Earnings. Rochester: University of Rochester, 1995.

BASU, S. 'The Conservatism Principle and the Asymmetric Timeliness of Earnings.' Journal of Accounting and Economics 25 (1997): 1-34.

BROOKS, L. D., and BUCKMASTER, D. A. 'Further Evidence of the Time Series Properties of Accounting Income.' The Journal of Finance 31 (1976): 1359-1373.

CAMPBELL, J. Y., and MANKIW, N. G. 'Permanent and Transitory Components in Macroeconomic Fluctuations.' American Economic Review 77 (1987): 111-117. 
CHEUNG, Y.-W., and CHINN, M. 'Further Investigation of the Uncertain Unit Root in GNP.' Journal of Business \& Economic Statistics 15 (1997): 68-75.

DICKEY, D. A., and FULLER, W. A. 'Distribution of the Estimators for Autoregressive Time Series With a Unit Root.' Journal of the American Statistical Association 74 (1979): 427-431.

DICKEY, D. A., and FULLER, W. A. 'Likelihood Ratio Tests for Autoregressive Time Series With a Unit Root.' Econometrica 49 (1981): 1057-1072.

ENDERS, W., and GRANGER, C. W. J. 'Unit Root Tests and Asymmetric Adjustment With an Example Using the Term Structure of Interest Rates.' Journal of Business \& Economic Statistics 16 (1998): 304-311.

FAMA, E. F., and FRENCH, K. R. 'Forecasting Profitability and Earnings.' Journal of Business 73 (2000): 161-176.

FINGER, C. A. 'The Ability of Earnings to Predict Future Earnings and Cash Flow.' Journal of Accounting Research 32 (1994): 210-223.

GIL-AlAnA, L. A., and PELÁEZ, R. F. 'The Persistence of Earnings Per Share.' Review of Quantitative Finance and Accounting 31 (2008): 425-439.

HADI, A. S. 'A Modification of a Method for the Detection of Outliers in Multivariate Samples.' Journal of the Royal Statistical Society, Series (B) 56 (1994): 393-396.

HAMILTON, J. D. Time Series Analysis. Princeton: Princeton University Press, 1994.

HAYN, C. 'The Information Content of Losses.' Journal of Accounting and Economics 20 (1995): 125-153.

LUETKEPOHL, H., and KRAETZIG, M. Applied Time Series Econometrics. Cambridge: Cambridge University Press, 2004.

NELSON, C., and PLOSSER, C. 'Trends and Random Walks in Macroeconomics Time Series: Some Evidence and Implications.' Journal of Monetary Economics 10 (1982): 139-162.

WHITE, H. 'A Heteroskedasticity-Consistent Covariance Matrix Estimator and a Direct Test 
for Heteroskedasticity.' Econometrica 48 (1980): 817-838.

WINDMEIJER, F. 'A Finite Sample Correction for the Variance of Linear Efficient Two-Step GMM Estimators.' Journal of Econometrics 126 (2005): 25-51. 